Corresponding author: mbainbridge@rchsd.org

(c) 2020 Sanford et al. This article is distributed under the terms of the Creative Commons

Attribution-NonCommercial License, which permits reuse and redistribution, except for commercial purposes, provided that the original author and source are credited.

Ontology terms: aspiration; bilateral vocal cord paresis; cardiorespiratory arrest; gastroesophageal reflux; laryngomalacia; neonatal hypoglycemia; respiratory failure

Published by Cold Spring Harbor Laboratory Press

doi:10.1101/mcs.a005611

\section{Postmortem diagnosis of PPA2- associated sudden cardiac death from dried blood spot in a neonate presenting with vocal cord paralysis}

\author{
Erica Sanford, ${ }^{1,2}$ Marilyn C. Jones, ${ }^{3}$ Matthew Brigger, ${ }^{4}$ Monia Hammer, ${ }^{1}$ \\ Lucia Giudugli, ${ }^{1}$ Stephen F. Kingsmore, ${ }^{1}$ David Dimmock, ${ }^{1}$ \\ and Matthew N. Bainbridge
}

\footnotetext{
${ }^{1}$ Rady Children's Institute of Genomic Medicine, ${ }^{2}$ Division of Pediatric Critical Care Medicine, Department of Pediatrics, ${ }^{3}$ Division of Genetics, Department of Pediatrics, University of California San Diego, La Jolla, California 92093, USA; ${ }^{4}$ Department of Otolaryngology, Rady Children's Hospital, San Diego, California 92123, USA
}

Abstract Biallelic variants in inorganic pyrophosphatase 2 (PPA2) are known to cause infantile sudden cardiac failure (OMIM \#617222), but relatively little is known about phenotypic variability of these patients prior to their death. We report a 5 -wk-old male with bilateral vocal cord paralysis and hypertension who had a sudden unexpected cardiac death. Subsequently, molecular autopsy via whole-genome sequencing from newborn dried blood spot identified compound heterozygous mutations in PPA2, with a paternally inherited, pathogenic missense variant (c.514G > A; p.Glu172Lys) and a novel, maternally inherited missense variant of uncertain significance (c.442A > T; p.Thr148Ser). This report expands the presenting phenotype of patients with PPA2 variants. It also highlights the utility of dried blood spots for postmortem molecular diagnosis.

[Supplemental material is available for this article.]

\section{INTRODUCTION}

Sudden unexpected death in infancy (SUDI) often remains unexplained despite comprehensive postmortem investigations. More recently, molecular autopsy has yielded answers for some of these patients (Torkamani et al. 2016). Variants that cause SUDI are typically very rare and may be dominant or recessive (Bainbridge 2020). Damaging variants in cardiacassociated genes have been found in 20\%-34\% of SUDI cases (Klaver et al. 2011; Hertz et al. 2016), including genes encoding cardiac ion channels and genes implicated in lethal cardiomyopathies (Klaver et al. 2011; Hertz et al. 2016). Next-generation sequencing (NGS) facilitates simultaneous analysis of a much larger number of genes, and this technologic advancement is likely responsible for the higher variant detection rate in cardiac genes in more recent publications (Hertz et al. 2016).

In 2016, Kennedy et al. and Guimier et al. separately identified biallelic missense variants in the gene PPA2 in several infants with sudden cardiac arrest (Guimier et al. 2016; Kennedy et al. 2016). PPA2 is a nuclear-encoded enzyme that has a critical role in mitochondrial maintenance and function (Guimier et al. 2016). The gene encodes an inorganic pyrophosphatase 2 that is responsible for hydrolyzing inorganic pyrophosphate (also known as diphosphate, PPi) into 


\begin{tabular}{lc}
\hline \multicolumn{2}{l}{ Table 1. Phenotypes previously associated with PPA2 deficiency and observed herein } \\
\hline Phenotype (HPO ID) & Present/Absent/Novel \\
\hline Bilateral vocal cord paresis (HP:0012822) & Novel \\
Laryngomalacia (HP:0001601) & Novel \\
Cardiac arrest (HP:0001695) & Present \\
Cardiomyopathy (HP:0001638) & Absent \\
Bradycardia (HP:0001662) & Present \\
Ventricular tachycardia (HP:0004756) & Present \\
Onset in early childhood (n.a.) & Present \\
Hypertension (HP:0000822) & Novel \\
Renal artery duplication (HP:0031922) & Novel \\
Vomiting (HP:0002013) & Present \\
Feeding difficulties in infancy (HP:0008872) & Present \\
Clinical seizures (HP:0001250) & Absent \\
Hypotonia (HP:0001290) & Absent \\
Lactic acidosis & Not assessed
\end{tabular}

two molecules of inorganic phosphate (also known as orthophosphate, Pi) (Mansurova 1989). This enzymatic reaction is essential for energy metabolism as it provides substrate for ATP synthesis (Mansurova 1989; Kennedy et al. 2016). It is also important for synthesis of DNA, RNA, protein, polysaccharides, and lipids (Mansurova 1989; Guimier et al. 2016).

To date there have been 19 patients described in the literature who suffered from sudden cardiac death attributed to disease-causing variants in PPA2 (https://omim.org/entry .617222, Guimier et al. 2016; Kennedy et al. 2016; Vasilescu et al. 2018; Phoon et al. 2020). In addition to the first patients identified by Kennedy et al. and Guimier et al., a Finnish cardiomyopathy cohort (KidCMP) of 66 children with severe cardiomyopathy found two siblings with compound heterozygous PPA2 variants who both died from rapidly progressive cardiomyopathy in infancy (Vasilescu et al. 2018). Additionally, in 2020, Phoon et al. reported two siblings who also died unexpectedly in infancy, and on postmortem analysis a molecular diagnosis of biallelic PPA2 variants was made (Phoon et al. 2020).

The majority of published patients were $<1 \mathrm{yr}$ of age at the time of cardiac arrest, with no comorbidities at their time of death (Table 1; Guimier et al. 2016; Kennedy et al. 2016; Vasilescu et al. 2018; Phoon et al. 2020). This report describes a 5-wk-old who was admitted and discharged from the Pediatric Intensive Care Unit (PICU) twice for symptoms of respiratory distress due to vocal cord paralysis, who then re-presented to the emergency room and had a cardiac arrest. Molecular autopsy via newborn dried blood spot identified compound heterozygous variants in PPA2. The novel clinical presentation in this infant potentially expands the spectrum of clinical phenotype associated with PPA2 variants.

\section{RESULTS}

\section{Clinical Presentation and Family History}

The male proband initially presented to his pediatrician at 3 wk of age for a weight check. He was noted to have fussiness, decreased oral intake, and emesis in the prior $3 \mathrm{~d}$. He had previously been diagnosed at $1 \mathrm{wk}$ of age with laryngomalacia by his pediatrician. He was sent to the emergency room for respiratory distress and then transferred to the PICU at Rady Children's Hospital, San Diego, for continuous positive airway pressure with 50\% oxygen 
(nasal CPAP). Review of systems and birth history were unremarkable. He was born at 39 wk to nonconsanguineous parents of European ancestry. Family history was notable for a paternal grandfather diagnosed with hypertrophic cardiomyopathy late in life. On admission to the PICU, radiography of the chest was within normal limits as was a chemistry panel. An echocardiogram was ordered to rule out a vascular ring, which was normal. Respiratory viral panel testing was positive for both rhinovirus and coronavirus, and his respiratory distress was attributed to viral bronchiolitis.

Because of persistent stridor, a bedside fiberoptic bronchoscopy was performed and revealed mild to moderate laryngomalacia as well as bilateral vocal cord hypomobility. The patient then went to the operating room for a more extensive airway evaluation, which confirmed bilateral vocal cord immobility. A dysphagia study showed aspiration of thin liquids, and his feeds were subsequently thickened. Brain magnetic resonance imaging was unremarkable. His oxygen requirement resolved and he was discharged home after $6 \mathrm{~d}$ in the hospital.

The patient returned the following day for recurrence of respiratory distress and was readmitted to the PICU. Oxygen support was initiated and a nasogastric tube was placed for feeding. Systemic hypertension was noted. Computed tomography angiogram of the chest and abdomen was remarkable for the finding of two left renal arteries without evidence of renal artery stenosis, and renal ultrasonography with Doppler waveform showed diminished diastolic flow consistent with elevated renal vascular resistance. The infant was started on amlodipine at the recommendation of the consulting nephrologist. Suture lateralization of the right vocal cord was performed to address persistent stridor. He was again weaned off of oxygen supplementation and discharged home on nasogastric tube feeds after $12 \mathrm{~d}$ in the hospital.

One day after his second discharge from the hospital, the patient returned to the emergency room following four episodes of vomiting initially thought to be due to malposition of the enteric tube. A radiograph was obtained that showed the tube terminating in the duodenum. During the period of observation in the emergency room, the patient had an unprovoked episode of unresponsiveness and cyanosis and subsequently had a bradycardic arrest. Chest compressions were initiated and the patient was intubated with an endotracheal tube. The underlying rhythm was primarily pulseless electrical activity (PEA), with one incidence of pulseless ventricular tachycardia, for which the patient was unsuccessfully defibrillated. Despite $1 \mathrm{~h}$ of cardiopulmonary resuscitation, including multiple doses of epinephrine, he was unable to be resuscitated.

At autopsy, the lungs were heavy (115 gm vs. expected $75 \mathrm{gm}$ ) with lymphocytic infiltration in the larynx and trachea, pulmonary congestion, and increased alveolar macrophages, consistent with a known history of silent aspiration. The liver weighed 227 gm (expected 140 $\mathrm{gm}$ ) and evidenced mild to moderate panacinar, anisomorphic, mostly macrovesicular steatosis. The heart was large (34 gm vs. expected $23 \mathrm{gm}$; Pryce et al. 2014) but was grossly and microscopically normal. There was no dilation or hypertrophy of the cardiac chambers. Skeletal muscle was microscopically normal. Hepatic steatosis was noted. There was no renal pathology. The brain was edematous but structurally normal.

\section{Genomic Analyses}

Proband sequencing (whole-genome sequencing [WGS]) was undertaken to determine a possible cause of death. This patient was found to be compound heterozygous for a paternally inherited, known pathogenic missense variant (c.514G > A; p.Glu172Lys; Chr 4: 106359121) and a maternally inherited, novel missense variant of uncertain significance (c.442A > T; p.Thr148Ser; Chr 4:106359193) in the PPA2 gene (Table 2). No additional sequence variants or copy-number variants (CNVs) were identified by WGS that could explain or be related to the patient's clinical presentation. 


\begin{tabular}{|c|c|c|c|c|c|c|c|c|c|}
\hline Gene & Chromosome & $\begin{array}{l}\text { HGVS DNA } \\
\text { reference }\end{array}$ & $\begin{array}{l}\text { HGVS } \\
\text { protein } \\
\text { reference }\end{array}$ & $\begin{array}{l}\text { Variant } \\
\text { type }\end{array}$ & $\begin{array}{l}\text { Predicted } \\
\text { effect }\end{array}$ & $\begin{array}{l}\text { dbSNP/ } \\
\text { dbVar ID }\end{array}$ & Genotype & ClinVar ID & $\begin{array}{l}\text { Parent of } \\
\text { origin }\end{array}$ \\
\hline PPA2 & $\begin{array}{l}\text { Chr 4:106359121 } \\
\text { (GRCh37) }\end{array}$ & $\begin{array}{c}\text { NM_176869.3: } \\
\text { c.514G>A }\end{array}$ & p.Glu172Lys & Missense & Substitution & rs146013446 & Heterozygous & SCV001250701.1 & Paternal \\
\hline PPA2 & $\begin{array}{l}\text { Chr 4:106359193 } \\
\text { (GRCh37) }\end{array}$ & $\begin{array}{c}\text { NM_176869.3: } \\
\text { c.442A>T }\end{array}$ & p.Thr148Ser & Missense & Substitution & None & Heterozygous & SCV001250702.1 & Maternal \\
\hline
\end{tabular}

The paternally inherited p.Glu172Lys variant in PPA2 has been previously reported as a compound heterozygous change in three individuals from two families with sudden unexpected cardiac arrest in infancy (Guimier et al. 2016) and in two individuals from one family who developed a rapidly progressive dilated cardiomyopathy and cardiac failure, with only a few days from disease onset to death (Vasilescu et al. 2018). This glutamine to lysine substitution is at a highly conserved residue and is predicted to disrupt at least three hydrogen bonds between interacting protein chains near the surface of the enzyme's active site and subsequently impair the enzymatic function of PPA2 (Guimier et al. 2016). This variant is present in the heterozygous state in the gnomAD population database at a frequency of $0.053 \%$ $(147 / 275080)$ overall and $0.095 \%$ in non-Finnish Europeans. Based on the available evidence, the c.514G > A (p.Glu172Lys) variant was classified as Pathogenic according to American College of Medical Genetics and Genomics (ACMG) and Association for Molecular Pathology (AMP) standards and guidelines (Richards et al. 2015). The specific scoring criteria used for classification are listed in Supplemental Table S1.

The maternally inherited c.442A $>\mathrm{T}$ is a novel missense variant. This variant has not been previously reported or functionally characterized in the literature to our knowledge. It is absent from the gnomAD population database and thus is presumed to be rare. The c.442A $>T$ (p.Thr148Ser) variant affects an amino acid that is conserved down to Arabidopsis thaliana and is predicted by multiple in silico tools to have a deleterious effect on protein function. The specific scoring criteria used for classification are listed in Supplemental Table S1. Results of protein sequence alignment from human to yeast shows remarkable sequence conservation of the Thr and its adjoining amino acids. This suggests an important evolutionary pressure to prevent a change of this Thr. We used three different algorithms to assess the mutations in the corresponding threonines (T100 in pdb code: 6C45 and T97 in pdb code: 4QLZ). These three methods of prediction are mCSM, SDM, and DUET (Topham et al. 1997; Worth et al. 2011; Pires et al. 2014). All three prediction tools point to a destabilizing effect of the missense change in both structures (Supplemental Table S2). Notably, the c.514G > A (p.Glu172Lys) was characterized as disruptive in a functional complementation assay in yeast where the human wild-type construct rescued a yeast growth defect, whereas the mutant $p$. Glu172Lys could not. Furthermore, the alteration was shown to affect the yeast mitochondrial function (Guimier et al. 2016). Based on the available evidence, the c.442A>T (p.Thr148Ser) variant was classified as a variant of uncertain significance.

\section{DISCUSSION}

NGS is increasingly being utilized for postmortem diagnosis of sudden unexplained death in infancy (Hertz et al. 2016). The genetic landscape for this condition is still being defined, in part because of the rare nature of this event and the novelty of the methodologic tools available (Vasilescu et al. 2018). Genes that were not previously analyzed in cases of sudden 
unexpected death in infancy have more recently been implicated as a probable cause of death (Hertz et al. 2016). The use of an NGS approach has shown diagnostic superiority compared with standard genetic testing and thus also makes for an ideal molecular autopsy tool, especially because of the ability to analyze recently discovered genes and genes not found in standard gene panels and to interrogate the genome for disorders that were not included in the premortem differential diagnosis because of an atypical or previously unrecognized presentation (Miller et al. 2015; Clark et al. 2018, 2019; Lionel et al. 2018; Vasilescu et al. 2018; Sanford et al. 2019). WGS is an ideal tool for the molecular autopsy as analysis is not limited by premortem differential diagnosis. However, it is worth noting that WGS does have its own limitations, including difficulty identifying trinucleotide repeat disorders as well as pathogenic deep intronic mutations (Smith et al. 2016).

We identified compound heterozygous mutations in PPA2 in a 5-wk-old infant who was admitted and discharged from the PICU twice for symptoms related to an unexplained vocal cord paralysis and then sustained a sudden unexpected cardiac arrest. The p.Glu172Lys variant identified in this patient has been reported as pathogenic for a sudden cardiac arrest in infancy and progressive lethal cardiomyopathy in multiple families (Guimier et al. 2016; Vasilescu et al. 2018), whereas this is the first report of the p.Thr148Ser variant to our knowledge and with the current available evidence it was classified as a variant of uncertain significance. The molecular diagnosis in this case was an unexpected finding, as the patient did not display any evidence of cardiac pathology (by telemetry or echocardiography) during two PICU admissions in the weeks prior to his death. This case underscores the knowledge that disease-causing PPA2 variants can cause sudden death in infancy without preceding symptoms (Phoon et al. 2020).

The p.Glu172Lys variant found in our patient has been previously reported as a compound heterozygous change in three individuals from two families with sudden unexpected cardiac arrest in infancy (Guimier et al. 2016) and in two brothers who developed a rapidly progressive dilated cardiomyopathy (Vasilescu et al. 2018). The siblings in the Vasilescu et al. cohort displayed a different phenotype than patients described by Guimier et al. (2016), Kennedy et al. (2016), and Phoon et al. (2020) as they had a few days between disease presentation and death (Vasilescu et al. 2018). The second brother was actually followed with serial echocardiograms, which were normal until the sudden manifestation of disease and rapid progression to cardiac death at 5 mo of age (Vasilescu et al. 2018). The patient in our case also had a documented normal echocardiogram $\sim 3 \mathrm{wk}$ prior to his death, although he experienced sudden death and no dilation was noted on cardiac autopsy.

As described previously, PPA2 variants occupy a range of the clinical spectrum, with sudden cardiac death documented from the neonatal period through adolescence, although the majority of patients died at $<1 \mathrm{yr}$ of age (Guimier et al. 2016; Kennedy et al. 2016; Vasilescu et al. 2018; Phoon et al. 2020). Two siblings of probands who suffered from sudden cardiac death were actually identified by genetic testing to have the same PPA2 variants, but received implantable defibrillators and were reportedly alive in their 30s with no events (Kennedy et al. 2016). Some cases of sudden cardiac arrest appeared to be associated with viral illness or alcohol ingestion, whereas for others there was no evidence of provocation (Kennedy et al. 2016; Phoon et al. 2020). Findings on necroscopy have ranged from nonspecific to cardiac inflammation, fibrosis, acute myocyte loss, and/or myocarditis (Kennedy et al. 2016; Vasilescu et al. 2018; Phoon et al. 2020). Similar to the two patients described by Phoon et al. the autopsy in our case was nonspecific (Phoon et al. 2020).

PPA2 was initially identified in yeast and was not associated with human disease until recently (Guimier et al. 2016; Kennedy et al. 2016; Vasilescu et al. 2018). The nuclear gene encodes an inorganic pyrophosphatase that functions within the mitochondrial matrix and hydrolyzes inorganic pyrophosphate (diphosphate) to two molecules of inorganic phosphate (orthophosphate), which is necessary for energy metabolism and numerous cellular reactions, 
including synthesis of DNA, RNA, proteins, polysaccharides, and lipids (Mansurova 1989; Guimier et al. 2016). Functional characterization in yeast has demonstrated its essential role in multiple cellular functions: In vitro PPA2 mutants, including the p.Glu172Lys variant, have demonstrated decreased oxidative respiration, reduced ATP synthesis, inability to maintain electrical potential across the mitochondrial inner membrane, and reduction in complex IV of the mitochondrial respiratory chain (Guimier et al. 2016; Kennedy et al. 2016; Vasilescu et al. 2018). Likewise, Eschericia coli expressing recombinant protein with the p.Glu172Lys variant had only $5 \%-10 \%$ residual activity (Kennedy et al. 2016). Studies of human cardiac and skeletal tissue have shown decreased mitochondrial complex I and IV in affected patients (Phoon et al. 2020). Examination of cardiac tissue on autopsy showed decreased levels of mitochondrial complex I subunit (two patients), decreased amount of PPA2 protein in heart tissue, and evidence indicating fewer mitochondria overall (Kennedy et al. 2016). Unfortunately, the mitochondria were not visualized on autopsy in the case presented here.

Further studies are needed to truly understand how the in vitro observations translate to cardiac dysfunction and ultimately cardiac death. Guimier hypothesized that the high energy needs of cardiac myocytes may preclude return of spontaneous circulation (ROSC) despite early and optimal resuscitation in patients with pathologic PPA2 variants, potentially because of an inability of the mitochondria to meet the energy demands of the cell (Guimier et al. 2016). This theory may be especially applicable to our patient as the majority of pediatric patients with in-hospital cardiac arrests do achieve ROSC (Sutton et al. 2018).

Our patient displayed a novel clinical presentation of bilateral vocal cord paresis. We suggest that this be considered as a potential phenotype expansion for PPA2 mutations. As noted, PPA2 variants result in a disorder affecting mitochondria. Several patients described had features consistent with classical descriptions of mitochondrial diseases, although nonspecific: hypotonia, myopathy, seizures, lactic acidosis, vomiting, and failure to thrive (Guimier et al. 2016; Kennedy et al. 2016). Vocal cord paralysis has been previously described in patients with a number of mitochondrial disorders (Supplemental Table S3), including KearnsSayre syndrome, Leigh disease, Brown-Vialetto-van Laere syndrome, Charcot-Marie-Tooth disease, and flavin adenine dinucleotide synthase (FADS) deficiency (Lin et al. 1999; Diamantopoulou et al. 2001; Sevilla et al. 2008; Chen et al. 2010; Ando et al. 2017; Thulasi et al. 2017; Yamada et al. 2019). Phenotypic expansion could have important implications for which patients receive more comprehensive genetic testing, such as WGS, and potentially a change in their clinical management-for example, implantation of a defibrillator, if appropriate, as in the cases of two affected siblings (Kennedy et al. 2016). However, the autopsy in this particular case was nonspecific and did not include an examination of the mitochondria. Additionally, the patient died at the age of $5 w k$, potentially prior to the manifestation of additional disease features. Thus, although we present this case as an interesting possibility, we are unable to say with certainty that bilateral vocal cord paresis is a phenotypic expansion of PPA2-related cardiac disease. More patients will need to be identified and more in vitro studies conducted to follow up on this potential association.

Molecular autopsy has the potential to influence family planning and familial screening and to more accurately define the genetic landscape of unexpected sudden death in infancy, regardless of premortem assumptions. This case illustrates that example, but more research is required to further elucidate the pathophysiology at play, and whether more comprehensive genetic screening of infants should be pursued earlier on.

\section{METHODS}

Parental consent was obtained for genome sequencing. Proband WGS was performed at Rady Children's Institute for Genomic Medicine as described (Torkamani et al. 2016; 


\begin{tabular}{ll}
\hline Table 3. Proband genome sequencing metrics & \\
\hline Metric & Value \\
\hline Read length & $2 \times 100 \mathrm{nt}$ \\
Mean coverage & 35 -fold \\
Nucleotide variants identified & $4,830,251$ \\
Variants with quality scores $>40$ & $93.5 \%$ \\
Coding nucleotide variants identified & 26,538 \\
Homozygous:heterozygous ratio of coding nucleotide variants & 0.64 \\
Transition to transversion ratio of coding variants & 2.9
\end{tabular}

Kingsmore et al. 2019). The newborn dried blood spot was released from the state laboratory for this purpose. Following DNA isolation from newborn dried blood spot (patient) and whole blood (parents), sequencing libraries were generated using the KAPA HyperPlus Library Prep kit (Roche) according to the manufacturer's instructions. Paired-end sequencing was performed on a NovaSeq 6000 instrument and S1 flow cell (Illumina). Read alignment to the reference human genome assembly GRCh37/hg19 and single-nucleotide variant (SNV)/ insertion-deletion (indel) calling was performed using the DRAGEN Bio-IT Platform (Illumina). CNV calling was performed using CNVnator and Manta (Kingsmore et al. 2019).

$\mathrm{SNV}$ s/indels and CNVs were annotated and analyzed using Fabric Enterprise version 6.5.2 (Fabric Genomics). The median genomic coverage was $35 \times$ with $93.3 \%$ of OMIM genes fully covered at $\geq 10 \times$ (Table 3 ).

Human Phenotype Ontology (HPO) terms used during variant interpretation included laryngomalacia (HP:0001601), bilateral vocal cord paresis (HP:0012822), aspiration (HP:0002835), hypoglycemia (HP:0001943), gastroesophageal reflux (HP:0002020), respiratory failure (HP:0002878), and cardiac arrest (HP:0001695). SNVs/indels were filtered to retain variants with allelic balance between 0.3 and 0.7 and allele frequency $<0.5 \%$ in the Genome Aggregation Database (gnomAD V2.1.1), prioritized by Phevor Gene Rank (Kingsmore et al. 2019), and classified according to ACMG and AMP standards and guidelines (Richards et al. 2015). CNVs were filtered to retain intragenic and intergenic variants shown to have a disease association or possibly associated with disease and classified according to ACMG and ClinGen recommendations (Riggs et al. 2020). Sequence variants of interest were orthogonally confirmed by Sanger sequencing, whereas CNVs of interest are typically confirmed by MLPA (none in this case).

\section{ADDITIONAL INFORMATION}

\section{Data Deposition and Access}

The causative variants have been submitted to ClinVar (https://www.ncbi.nlm.nih.gov/ clinvar/) with accession numbers SCV001250701.1 and SCV001250702.1. Raw sequencing data could not be deposited because patient consent was not obtained.

\section{Ethics Statement}

Informed and signed consent forms were obtained for all sequenced individuals in this study. The project is approved by the Institutional Review Board of the University of California at San Diego under protocol \#20172719 and has received nonsignificant risk status in a preInvestigational Device Exemption submission to the Food and Drug Administration. 
Competing Interest Statement

The authors have declared no competing interest.

Referees

Colin Phoon

Anonymous

Received May 20, 2020; accepted in revised form August 12, 2020.

\section{Acknowledgments}

We are extremely thankful for the family's willingness to participate in this study.

\section{Author Contributions}

M.N.B. conceived of the study. E.S., L.G., and M.H. wrote the manuscript. All authors reviewed and edited the manuscript.

\section{Funding}

Funding for this study was provided in part by the National Heart, Lung, and Blood Institute (NHLBI) (R01HL145175-02) and by the Scripps Research Institute (5 UL1 TR002550).

\section{REFERENCES}

Ando M, Hashiguchi A, Okamoto Y, Yoshimura A, Hiramatsu Y, Yuan J, Higuchi Y, Mitsui J, Ishiura H, Umemura A, et al. 2017. Clinical and genetic diversities of Charcot-Marie-Tooth disease with MFN2 mutations in a large case study. J Peripher Nerv Syst 22: 191-199. doi:10.1111/jns.12228

Bainbridge MN. 2020. Determining the incidence of rare diseases. Hum Genet 139: 569-574. doi:10.1007/ s00439-020-02135-5

Chen DH, Sul Y, Weiss M, Hillel A, Lipe H, Wolff J, Matsushita M, Raskind W, Bird T. 2010. CMT2C with vocal cord paresis associated with short stature and mutations in the TRPV4 gene. Neurology 75: 1968-1975. doi:10.1212/WNL.0b013e3181ffe4bb

Clark MM, Stark Z, Farnaes L, Tan TY, White SM, Dimmock D, Kingsmore SF. 2018. Meta-analysis of the diagnostic and clinical utility of genome and exome sequencing and chromosomal microarray in children with suspected genetic diseases. NPJ Genom Med 3: 16. doi:10.1038/s41525-018-0053-8

Clark M, Hildreth A, Batalov S, Ding Y, Chowdhury S, Watkins K, Ellsworth K, Camp B, Kint Cl, Yacuobian C, et al. 2019. Diagnosis of genetic diseases in seriously ill children by rapid whole-genome sequencing and automated phenotyping and interpretation. Sci Transl Med 11: eaat6177. doi:10.1126/scitranslmed .aat6177

Diamantopoulou P, Ward VM, Harries ML. 2001. Kearns-Sayre syndrome: presenting with vocal fold palsy. J Laryngol Otol 115: 1021-1022. doi:10.1258/0022215011909675

Guimier A, Gordon CT, Godard F, Ravenscroft G, Oufadem M, Vasnier C, Rambaud C, Nitschke P, Bole-Feysot C, Masson C, et al. 2016. Biallelic PPA2 mutations cause sudden unexpected cardiac arrest in infancy. Am J Hum Genet 99: 666-673. doi:10.1016/j.ajhg.2016.06.021

Hertz CL, Christiansen SL, Larsen MK, Dahl M, Ferrero-Miliani L, Weeke PE, Pedersen O, Hansen T, Grarup N Ottesen GL, et al. 2016. Genetic investigations of sudden unexpected deaths in infancy using next-generation sequencing of 100 genes associated with cardiac diseases. Eur J Hum Genet 24: 817-822. doi:10 .1038/ejhg.2015.198

Kennedy H, Haack TB, Hartill V, Matakovic L, Regula Baumgartner E, Potter H, Mackay R, Alston CL, O'Sullivan $S$, McFarland R, et al. 2016. Sudden cardiac death due to deficiency of the mitochondrial inorganic pyrophosphatase PPA2. Am J Hum Genet 99: 674-682. doi:10.1016/j.ajhg.2016.06.027

Kingsmore SF, Cakici JA, Clark MM, Gaughran M, Feddock M, Batalov S, Bainbridge MN, Carroll J, Caylor SA, Clarke $C$, et al. 2019. A randomized, controlled trial of the analytic and diagnostic performance of singleton and trio, rapid genome and exome sequencing in ill infants. Am J Hum Genet 105: 719-733. doi:10.1016/j .ajhg.2019.08.009

Klaver EC, Verslujis GM, Wilders R. 2011. Cardiac ion channel mutations in the sudden infant death syndrome. Int J Cardiol 152: 162-170. doi:10.1016/j.ijcard.2010.12.051

Lin YC, Lee WT, Wang PJ, Shen YZ. 1999. Vocal cord paralysis and hypoventilation in a patient with suspected Leigh disease. Pediatr Neurol 20: 223-225. doi:10.1016/S0887-8994(98)00137-4

Lionel AC, Costain G, Monfared N, Walker S, Reuter MS, Hosseini SM, Thiruvahindrapuram B, Merico D, Jobling R, Nalpathamkalam T, et al. 2018. Improved diagnostic yield compared with targeted gene sequencing panels suggests a role for whole-genome sequencing as a first-tier genetic test. Genet Med 20: 435-443. doi:10.1038/gim.2017.119

Mansurova SE. 1989. Inorganic pyrophosphate in mitochondrial metabolism. Biochim Biophys Acta 977: 237247. doi:10.1016/s0005-2728(89)80078-7 
Miller NA, Farrow EG, Gibson M, Willig LK, Twist G, Yoo B, Marrs T, Corder S, Krivohlavek L, Walter A, et al. 2015. A 26-hour system of highly sensitive whole genome sequencing for emergency management of genetic diseases. Genome Med 7: 100. doi:10.1186/s13073-015-0221-8

Phoon CKL, Halvorsen M, Goldstein DB, Rabin R, Cecchin F, Crandall L, Devinsky O. 2020. Sudden unexpected death in asymptomatic infants due to PPA2 variants. Mol Genet Genomic Med 8: e1008. doi:10.1002/ mgg3.1008

Pires DE, Ascher DB, Blundell TL. 2014. DUET: a server for predicting effects of mutations on protein stability using an integrated computational approach. Nucleic Acids Res 42: W314-W319. doi:10.1093/nar/ gku411

Pryce JW, Bamber AR, Ashworth MT, Kiho L, Malone M, Sebire NJ. 2014. Reference ranges for organ weights of infants at autopsy: results of $>1,000$ consecutive cases from a single centre. BMC Clin Pathol 14: 18 . doi:10.1186/1472-6890-14-18

Richards S, Aziz N, Bale S, Bick D, Das S, Gastier-Foster J, Grody WW, Hegde M, Lyon E, Spector E, et al. 2015 Standards and guidelines for the interpretation of sequence variants: a joint consensus recommendation of the American College of Medical Genetics and Genomics and the Association for Molecular Pathology. Genet Med 17: 405-424. doi:10.1038/gim.2015.30

Riggs ER, Andersen EF, Cherry AM, Kantarci S, Kearney H, Patel A, Raca G, Ritter DI, South ST, Thorland EC, et al. 2020. Technical standards for the interpretation and reporting of constitutional copy-number variants: a joint consensus recommendation of the American College of Medical Genetics and Genomics (ACMG) and the Clinical Genome Resource (ClinGen). Genet Med 22: 245-257. doi:10.1038/s41436-019-0686-8

Sanford EF, Clark MM, Farnaes L, Williams MR, Perry JC, Ingulli EG, Sweeney NM, Doshi A, Gold JJ, Briggs B, et al. 2019. Rapid whole genome sequencing has clinical utility in children in the PICU. Pediatr Crit Care Med 20: 1007-1020. doi:10.1097/PCC.0000000000002056

Sevilla T, Jaijo T, Nauffal D, Collado D, Chumillas MJ, Vilchez J, Muelas N, Bataller L, Domenech R, Espinos C, et al. 2008. Vocal cord paresis and diaphragmatic dysfunction are severe and frequent symptoms of GDAP1-associated neuropathy. Brain 131: 3051-3061. doi:10.1093/brain/awn228

Smith LD, Willig LK, Kingsmore SF. 2016. Whole-exome sequencing and whole-genome sequencing in critically ill neonates suspected to have single-gene disorders. Cold Spring Harb Perspect Med 6: 1-15. doi:10 $.1101 /$ cshperspect.a023168

Sutton RM, Reeder RW, Landis W, Meert KL, Yates AR, Berger JT, Newth CJ, Carcillo JA, McQuillen PS, Harrison RE, et al. 2018. Chest compression rates and pediatric in-hospital cardiac arrest survival outcomes. Resuscitation 130: 159-166. doi:10.1016/j.resuscitation.2018.07.015

Thulasi V, Veerapandiyan A, Pletcher BA, Tong CM, Ming X. 2017. A case of Brown-Vialetto-van Laere syndrome due to a novel mutation in SLC52A3 gene: clinical course and response to riboflavin. Child Neurol 4: 2329048X17725610. doi:10.1177/2329048X17725610

Topham CM, Srinivasan N, Blundell TL. 1997. Prediction of the stability of protein mutants based on structural environment-dependent amino acid substitution and propensity tables. Protein Eng 10: 7-21. doi:10 .1093/protein/10.1.7

Torkamani A, Muse ED, Spencer EG, Rueda M, Wagner GN, Lucas JR, Topol EJ. 2016. Molecular autopsy for sudden unexpected death. J Am Med Assoc 316: 1492-1494. doi:10.1001/jama.2016.11445

Vasilescu C, Ojala TH, Brilhante V, Ojanen S, Hinterding HM, Palin E, Alastalo TP, Koskenvuo J, Hiippala A,

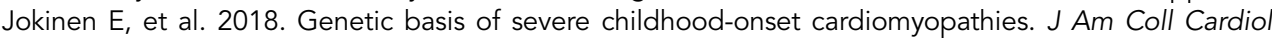
72: 2324-2338. doi:10.1016/j.jacc.2018.08.2171

Worth CL, Preissner R, Blundell TL. 2011. SDM-a server for predicting effects of mutations on protein stability and malfunction. Nucleic Acids Res 39: W215-W222. doi:10.1093/nar/gkr363

Yamada K, Ito M, Kobayashi H, Hasegawa Y, Fukuda S, Yamaguchi S, Taketani T. 2019. Flavin adenine dinucleotide synthase deficiency due to FLAD1 mutation presenting as multiple acyl-CoA dehydrogenation deficiency-like disease: a case report. Brain Dev 41: 638-642. doi:10.1016/j.braindev.2019.04.002 


\section{COLD SPRING HARBOR Molecular Case Studies}

\section{Postmortem diagnosis of PPA2-associated sudden cardiac death from dried blood spot in a neonate presenting with vocal cord paralysis}

Erica Sanford, Marilyn C. Jones, Matthew Brigger, et al.

Cold Spring Harb Mol Case Stud 2020, 6: a005611

Access the most recent version at doi:10.1101/mcs.a005611

Supplementary http://molecularcasestudies.cshlp.org/content/suppl/2020/10/07/mcs.a005611.D
Material

References This article cites 30 articles, 2 of which can be accessed free at:

http://molecularcasestudies.cshlp.org/content/6/5/a005611.full.html\#ref-list-1

License This article is distributed under the terms of the Creative Commons Attribution-NonCommercial License, which permits reuse and redistribution, except for commercial purposes, provided that the original author and source are credited.

Email Alerting Receive free email alerts when new articles cite this article - sign up in the box at the Service top right corner of the article or click here. 